\title{
Les développements éoliens au large des côtes françaises Introduction à la journée technique du 06 décembre 2018
}

\author{
Patrick Berthelot ${ }^{1}$, Alain Puech ${ }^{2, \star}$ et Françoise Ropers ${ }^{3, \text { a }}$ \\ ${ }^{1}$ Bureau Veritas/PBe ConseilS, Paris, France \\ 2 Fugro GeoConsulting, Nanterre, France \\ 3 Terrasol, Paris, France
}

\begin{abstract}
Résumé - Le développement de fermes éoliennes au large des côtes françaises est entré dans une phase active. Cette communication résume brièvement les projets en cours et l'état du marché fin 2018. Pour pallier l'absence de documents normatifs nationaux, le CFMS a mis en place en 2013 un Groupe de Travail ayant pour mission d'élaborer des «Recommandations pour la conception et le dimensionnement des fondations d'éoliennes offshore». Le cadre du document présenté le 06 décembre à la communauté géotechnique française est rappelé et ses spécificités sont soulignées. Une attention particulière a été portée aux conditions spécifiques des sols et roches rencontrés sur le plateau continental français ainsi qu'au dimensionnement de pieux forés et cimentés qui est peu couvert par les réglementations internationales en usage dans l'industrie offshore. L'après-midi du 06 décembre était consacrée à des communications techniques sur trois projets majeurs de R\&D: PISA, ALPACA et SOLCYP+.
\end{abstract}

Mots clés : éolien offshore / côtes françaises / développements en cours

\begin{abstract}
Wind farm developments offshore France
Introduction to the technical day of 06 December 2018. The development of wind farms offshore France has entered into an active phase. This paper makes a brief review of ongoing projects and outlines the main features of the market by end 2018. Considering the absence of national normative documents, the CFMS has set up in 2013 a Working Group aimed at elaborating "Recommendations for planning and designing offshore wind turbine foundations". The framework of this document presented on December 06, 2018 to the French geotechnical community is outlined and its specificities are highlighted. A particular attention is paid to the peculiar conditions of the soils and rocks encountered on the French continental shelf and to the design of drilled and grouted piles which is poorly addressed in the international codes in use in the offshore industry. In the afternoon of the 06th December, the focus was put on three major R\&D projects: PISA, ALPACA and SOLCYP+.
\end{abstract}

Keywords: offshore wind farms / French coasts / ongoing developments

\section{Marché de l'éolien offshore français}

Dans le cadre du Grenelle de l'environnement et du paquet «énergie-climat » la France avait pris l'engagement d'installer 6000 MW d'éolien en mer posé à l'horizon 2020 et d'engager 9000 MW avant 2023.

Ces objectifs annoncés seront loin d'être tenus si on en croit les projets réellement engagés et les retards accumulés ne serait-ce que du fait de l'inefficacité des procédures d'instruction et des recours juridiques à répétition.

\footnotetext{
^ Auteur de correspondance : a.puech@fugro.com

${ }^{a}$ Consultante auprès de Terrasol.
}

Le premier appel d'offres a été lancé par la puissance publique en 2011. Il portait sur un potentiel de 2500 à $3000 \mathrm{MW}$ répartis sur 5 sites. À l'issue du processus, seulement 4 sites ont été attribués pour une puissance de $2100 \mathrm{MW}$ (environ 450 à $500 \mathrm{MW}$ par site) : Saint-Nazaire, St Brieuc, Courseulles-sur-Mer et Fécamp. Leur mise en service était prévue entre 2019 et 2021 . À ce jour aucun de ces projets n'a obtenu une autorisation finale d'investissement.

Un deuxième Appel d'Offres a permis de confirmer en 2013 l'attribution du cinquième site (Le Tréport) et d'un sixième site dit Ile d'Yeu-Noirmoutier. Le site de Dunkerque a été attribué dans le cadre du troisième appel d'offres de 2018. La zone d'Oléron est retenue pour un appel d'offres à venir.

L'implantation des sites attribués est indiquée sur la figure 1. Le tableau 1 récapitule les sites actuellement 


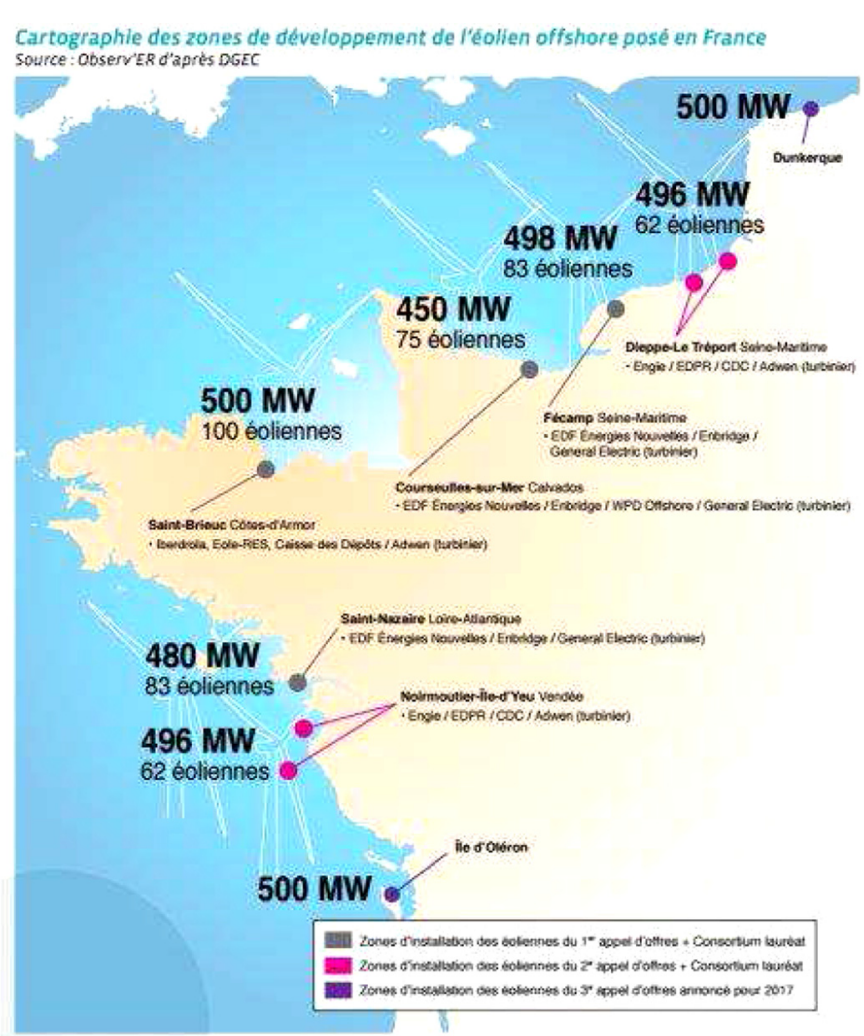

Fig. 1. Sites des projets d'éoliennes en mer sur les côtes Françaises ayant fait l'objet d'appels d'offres (Sources : eoliennes-mer.fr).

Fig. 1. Locations of ongoing windfarm projects offshore France.

envisagés pour le développement de fermes d'éoliennes posées. Il précise le nombre d'éoliennes par site (dans la fourchette $62-83$ ) et la puissance des turbines retenues $(5,6$ ou $8 \mathrm{MW})$. La puissance unitaire tend à croître et on parle déjà de $10 \mathrm{MW}$ pour les projets à venir.

Malgré une renégociation du prix d'achat de l'électricité produite, celui-ci reste élevé à $150 € / \mathrm{MWh}$. Par comparaison, au Royaume Uni, où une politique de développement volontariste a permis une expansion fulgurante de l'industrie éolienne en mer, le prix du MWh est tombé aux alentours de $50-55 €$, inférieur au prix du nucléaire négocié sur les nouvelles centrales (Hinkley Point).

\section{Les fondations des éoliennes posées en mer}

Trois types de fondations sont couramment utilisés pour les éoliennes posées en mer: les monopieux, les pieux et les embases gravitaires. Chacune de ces fondations est associée à un type particulier de sous-structure, respectivement monopode, structure métallique de type réticulé (jacket) ou tripode, et colonne en béton armé et précontraint ballastée. La fondation sur monopieu est de loin la plus couramment utilisée à ce jour dans des profondeurs d'eau inférieures à la cinquantaine de mètres.

Les figures 2-4 illustrent chaque type de fondation.

Pour une description plus complète de ces fondations on pourra se reporter aux «Recommandations pour la conception et le dimensionnement des fondations d'éoliennes offshore » du CFMS (Comité Français de Mécanique des Sols et de Géotechnique) introduites en section 5 ci-après.

Les sites attribués font appel aux trois types de fondations usuels :

- monopieux pour les sites de St Nazaire et Courseulles-surMer ;

- jackets sur pieux pour les sites de St Brieuc, Le Tréport et Yeu-Noirmoutier;

- embases gravitaires pour Fécamp.

\section{Les projets d'éoliennes flottantes}

L'éolien flottant est encore loin d'être une industrie mature. En France cinq projets pilotes sont en cours de développement. Ils sont listés dans le tableau 2.

Ces projets comportent l'installation d'une à quatre éoliennes de puissances comprises entre 2 et $8 \mathrm{MW}$.

À ce jour seule l'éolienne Floatgen est installée au Croisic.

Les autres éoliennes sont prévues pour être ancrées par lignes caténaires et ancres draguées (e.g. Fig. 5) à l'exception des éoliennes du projet Provence Grand Large qui seront sur lignes tendues reliées à des ancres mises en place par succion (Fig. 6).

\section{Les sols du plateau continental français}

Les sols rencontrés sur le plateau continental français (notamment côtes Atlantiques et Manche) peuvent être classés en trois grandes catégories :

- les sols usuellement rencontrés en mer: sables siliceux, argiles normalement consolidées à fortement surconsolidées. Le comportement de ces matériaux est réputé connu et les procédures de dimensionnement des fondations sont bien documentées dans les codes couramment utilisés dans l'industrie offshore, par exemple DNV GL-OS-J126 (2016);

- les sols déjà rencontrés sur d'autres sites de développement éolien mais dont le comportement est réputé mal connu et difficilement prévisible: il s'agit notamment des craies présentes sur de nombreux sites au large du Royaume Uni et en Mer Baltique. Elles font l'objet d'actions de recherche de grande ampleur. On citera les essais in situ en vrai grandeur réalisés sur le champ de Wikinger ainsi que les projets de recherche ALPACA au Royaume Uni et SOLCYP+ en France. Plusieurs présentations techniques leur ont été consacrées lors de la journée du 06 décembre 2018 (Dupla et al., 2019; Jardine et al., 2019). On notera une difficulté supplémentaire qui tient à une plus forte teneur en silex des craies rencontrées du côté français de la Manche;

- les sols non rencontrés jusqu'à présent dans le cadre de développements éoliens offshore. Il s'agit de:

- sables carbonatés plus ou moins cimentés et calcarénites avec des résistances à la compression simple typiquement inférieures à $5 \mathrm{MPa}$. L'expérience acquise par l'industrie pétrolière offshore dans diverses régions du globe (Golfe Arabo-persique, Australie, etc.) peut être utilisée ;

- Marnes. Elles sont fréquemment rencontrées à terre sur le sol national et traitées dans les normes françaises (dont 
Tableau 1. Caractéristiques et état d'avancement des projets éoliens offshore français à fin 2018 .

Table 1. Characteristics and status of offshore windfarm projects offshore France by end 2018.

\begin{tabular}{llll}
\hline Projet & Reconnaissance préliminaire & Reconnaissance finale & Dimensionnement \\
\hline Fécamp $(83 \times 5 \mathrm{MW})$ Embases gravitaires & $\mathrm{X}$ & $\mathrm{X}$ & $\mathrm{D}$ \\
St Nazaire $(80 \times 5 \mathrm{MW})$ Monopieux & $\mathrm{X}$ & $\mathrm{X}$ & $\mathrm{D}$ \\
Courseulles $(75 \times 5 \mathrm{MW})$ Monopieux & $\mathrm{X}$ & $\mathrm{X}$ & $\mathrm{P}$ \\
St Brieuc $(62 \times 8 \mathrm{MW})$ Jackets sur pieux & $\mathrm{X}$ & $\mathrm{X}$ & $\mathrm{P}$ \\
Le Tréport $(62 \times 6 \mathrm{MW})$ Jackets sur pieux & $\mathrm{X}$ & $\mathrm{O}$ & $\mathrm{P}$ \\
Yeu-Noirmoutier $(62 \times 6 \mathrm{MW})$ Jackets sur pieux & $\mathrm{X}$ & Appel d'Offres en cours \\
Dunkerque & & Appel d'Offres à venir \\
Oléron & & - \\
\hline
\end{tabular}

$\mathrm{X}=$ fait $; \mathrm{O}=$ en cours $2018 ; \mathrm{P}=$ préliminaire $; \mathrm{D}=$ dimensionnement détaillé en cours.

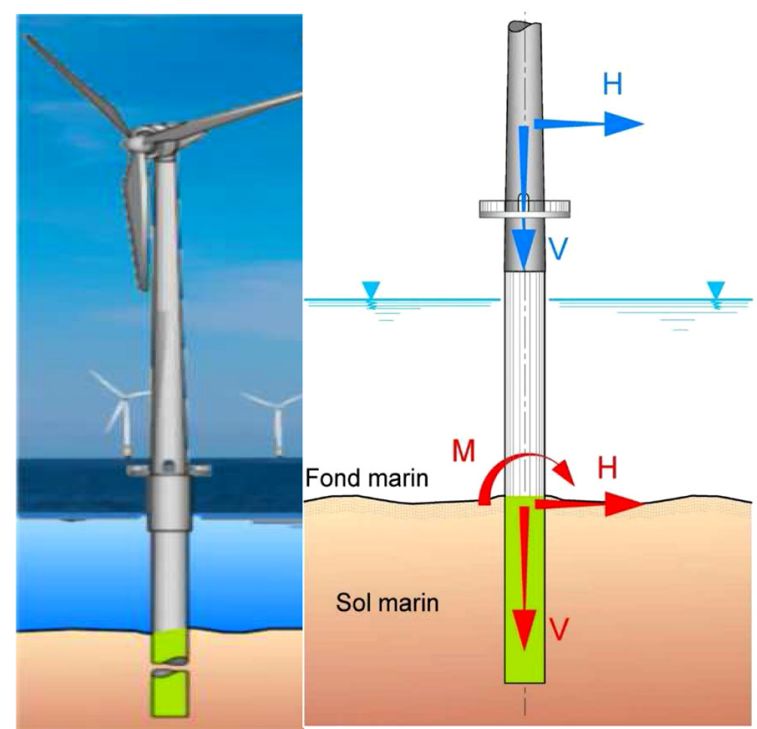

Fig. 2. Fondation sur monopieu-Principe.

Fig. 2. Monopile foundation-Principle.

NF P 94-262) mais ne sont signalées dans aucun document normatif applicable dans l'industrie offshore;

- roches moyennement dures à dures avec des résistances à la compression simple comprises entre 10 et $100 \mathrm{MPa}$. Ces matériaux se rencontrent avec des degrés de fracturation et d'altération variables. Là encore ce type de matériau n'est pas considéré dans la réglementation offshore.

Le recours à des pieux forés sera souvent nécessaire dans les calcarénites, marnes et roches tendres. Les recommandations à destination des fondations d'ouvrages offshore sont quasi inexistantes ou très succinctes pour ce type de fondations. Par opposition l'expérience terrestre française est bien documentée.

\section{Recommandations pour la conception et le dimensionnement des fondations d'éoliennes offshore}

En 2012 ont été publiées sous l'égide du Comité Français de Mécanique des Sols et de Géotechnique des «Recom- mandations sur la conception, le calcul, l'exécution et le contrôle des fondations d'éoliennes » dédiées aux éoliennes terrestres. Elles ont été publiées dans la RFG n ${ }^{\circ} 138-139$ en 2012. Afin de suivre les évolutions technologiques et la demande du marché des énergies renouvelables, le Comité Français de Mécanique des Sols et de Géotechnique (CFMS) a décidé dès 2013 la création d'un nouveau groupe de travail pour traiter des aspects géotechniques des fondations d'éoliennes offshore.

Finalisées en 2018, ces «Recommandations pour la conception et le dimensionnement des fondations d'éoliennes offshore» ont pour vocation de permettre aux différents concepteurs de pallier l'absence de documents normatifs ou de textes réglementaires nationaux concernant la conception et la réalisation des fondations des ouvrages offshore dans les eaux territoriales françaises (hors charges sismiques).

Pendant près de cinq ans, une vingtaine de personnes représentant l'ensemble de la profession (Maîtres d'ouvrages, Maîtres d'Oeuvre, ingénieries géotechniques, constructeurs, entreprises, institutionnels) a participé aux réunions des différents sous-groupes ainsi qu'aux réunions plénières.

Les «Recommandations pour la conception et le dimensionnement des fondations d'éoliennes offshore» traitent exclusivement des fondations d'éoliennes fixes et des types les plus fréquemment rencontrés à ce jour à savoir les fondations sur monopieux, les fondations sur pieux et les fondations sur embases gravitaires.

Les Recommandations s'appuient sur et viennent en complément des normes internationales applicables à l'industrie offshore et plus spécifiquement aux éoliennes offshore : IEC 61400-1; IEC 61400-3 et DNVGL-RP-C212; DNVGLST-0126; DNVGL-ST-0145; DNVGL-ST-0437 (avec IEC : International Electrotechnical Commission et DNVGL: Det Norske Veritas-Germanischer Lloyd).

Les particularités essentielles de ce document résident dans les points forts suivants :

- les études de terrains nécessaires à la conception et au dimensionnement des ouvrages offshore ont été traitées de manière approfondie ;

- des catégories de sols spécifiques sont rencontrées sur le plateau continental français (craies, marnes, calcarénites). Les codes internationaux se limitant aux sols conventionnels (sables siliceux, argiles), une attention particulière a été portée aux matériaux à teneur en calcaire significative ; 

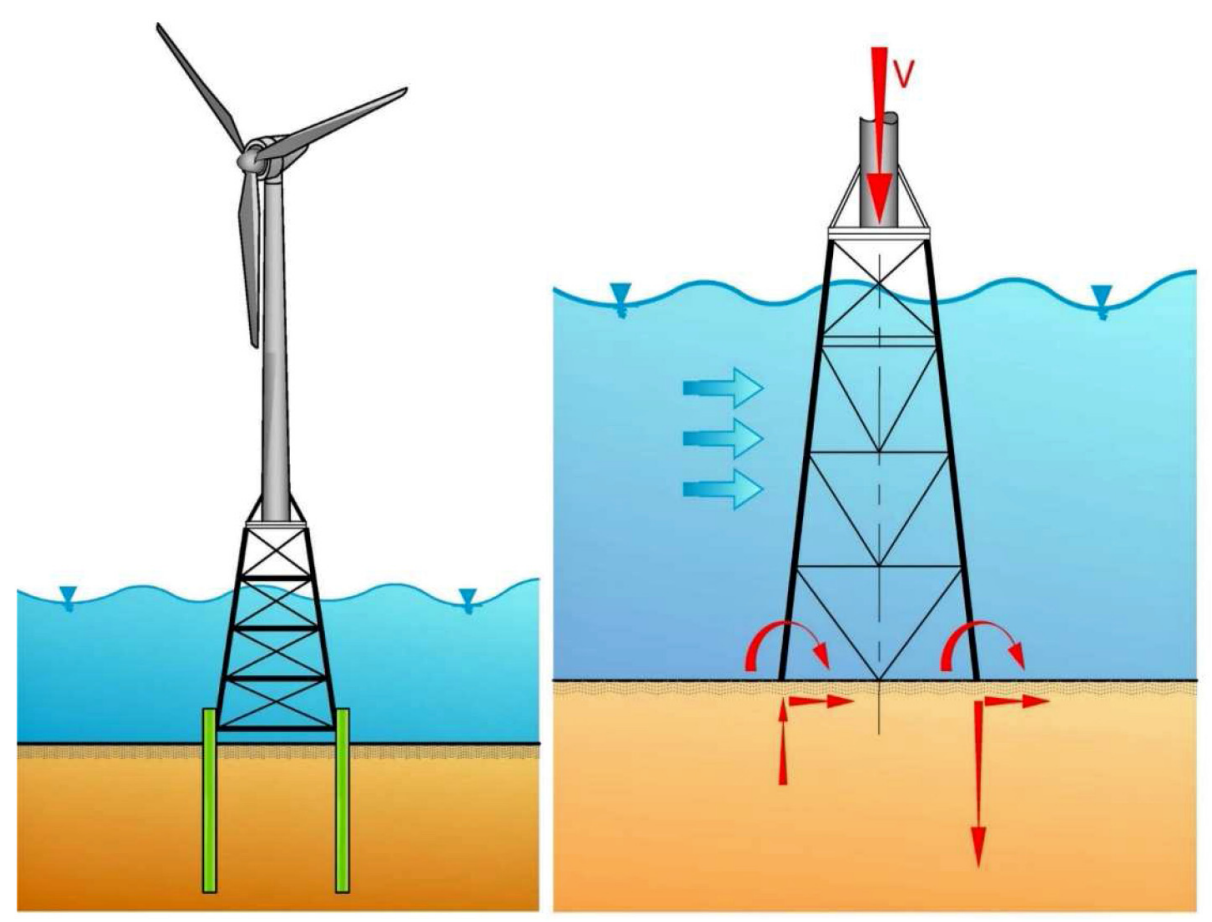

Fig. 3. Fondation sur jacket et pieux - Principe.

Fig. 3. Foundation on jacket and piles-Principle.
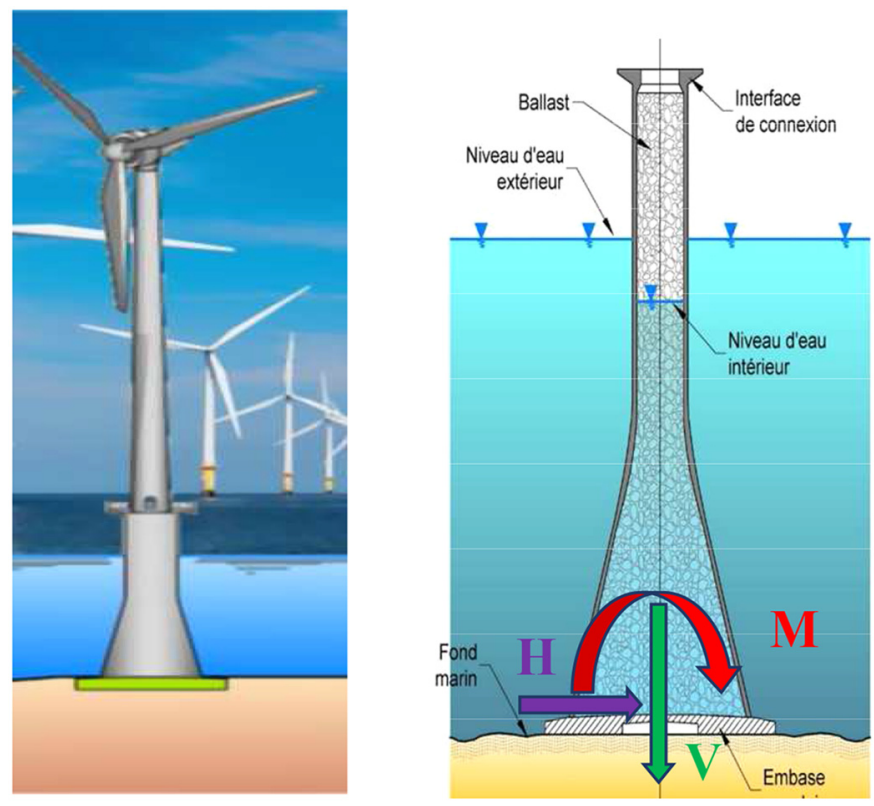

Fig. 4. Fondation sur embase gravitaire-Principe.

Fig. 4. Gravity base foundation-Principle.

- plusieurs sites de fermes éoliennes sont concernés par des substratums rocheux, dans lesquels le recours aux pieux forés et cimentés sera incontournable. Ce type de fondation n'est que très sommairement abordé dans les codes internationaux. En revanche, il est communément utilisé en géotechnique terrestre. Il est apparu indispensable d'établir un pont entre les pratiques de l'industrie offshore et le savoir-faire français dans le domaine des pieux forés. Une harmonisation en termes de sécurité a été nécessaire ;

- le traitement des charges cycliques a été explicité. Les apports du projet SOLCYP ont été intégrés notamment en ce qui concerne le traitement des charges cycliques et les méthodologies de dimensionnement des pieux.

Le document final de 250 pages se compose de 11 chapitres et 3 annexes :

- Avant propos

- Chapitre 1: Introduction

- Chapitre 2: Référentiel

- Chapitre 3: Conditions de sites

- Chapitre 4: Charges et sollicitations

- Chapitre 5: Études de terrains

- Chapitre 6: Paramètres de sol et profils de dimensionnement

- Chapitre 7: Sollicitations et vérifications

- Chapitre 8: Monopieux

- Chapitre 9: Pieux

- Chapitre 10: Embases gravitaires

- Chapitre 11: Affouillement et mobilité sédimentaire

- Annexe A: Tableau des charges

- Annexe B : Embases annulaires

- Annexe C: Exemple de modélisation d'une fondation gravitaire par éléments finis

La version 2.0 des « Recommandations pour la conception et le dimensionnement des fondations d'éoliennes offshore» est disponible sur le site du CFMS : www.cfms-sols.org. Elle est également publiée sous la forme d'un numéro spécial $\mathrm{N}^{\mathrm{o}} 157$ de la Revue Française de Géotechnique (RFG). 
Tableau 2. Eoliennes flottantes. Projets pilotes.

Table 2. Floating wind turbines. Experimental projects.

\begin{tabular}{llll}
\hline Projet & Reconnaissance préliminaire & Reconnaissance complète & Dimensionnement \\
\hline $\begin{array}{l}\text { Floatgen, Le Croisic }(1 \times 2 \mathrm{MW}) \\
\text { Ancrage caténaire }\end{array}$ & $\mathrm{X}$ & $\mathrm{X}$ & $\mathrm{X}$ installée \\
Gruissan $(4 \times 6 \mathrm{MW})$ Ancrage caténaire & $\mathrm{X}$ & & $\mathrm{P}$ \\
Leucate $(4 \times 6 \mathrm{MW})$ Ancrage caténaire & $\mathrm{O}$ & $\mathrm{P}$ \\
Groix $(4 \times 6 \mathrm{MW})$ Ancrage caténaire & $\mathrm{X}$ & $\mathrm{X}$ & $\mathrm{P}$ \\
Provence Grand Large $(3 \times 8 \mathrm{MW})$ Ancrage tendu & $\mathrm{X}$ & $\mathrm{D}$ \\
\hline
\end{tabular}

$\mathrm{X}=$ fait $; \mathrm{O}=$ en cours $2018 ; \mathrm{P}=$ préliminaire $; \mathrm{D}=$ dimensionnement détaillé en cours.

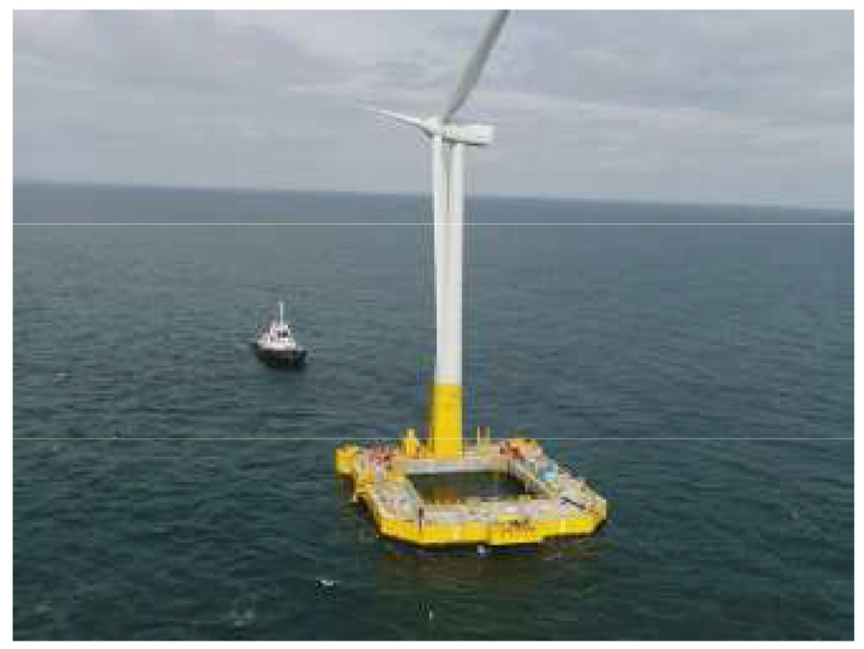

Fig. 5. Eolienne flottante de type Bouygues - Ideol.

Fig. 5. Floating wind turbine of the Bouygues-Ideol type.

Le document est en cours de traduction en langue anglaise et sera disponible sur les mêmes supports au cours du deuxième semestre 2019.

\section{Organisation de la journée de restitution du 06 décembre 2018}

Le CFMS a organisé le 06 décembre 2018 une Journée Technique consacrée aux fondations d'éoliennes offshore.

La matinée était réservée à la présentation des «Recommandations pour la conception et le dimensionnement des fondations d'éoliennes offshore».

L'après-midi était ouverte à des présentations techniques sur l'avancement et les premiers résultats de trois projets de recherche et développement particulièrement significatifs pour l'avenir de l'industrie éolienne offshore:

- le projet PISA dédié au comportement des monopieux sous chargements latéraux;

- le projet ALPACA traitant du comportement des pieux battus dans la craie;

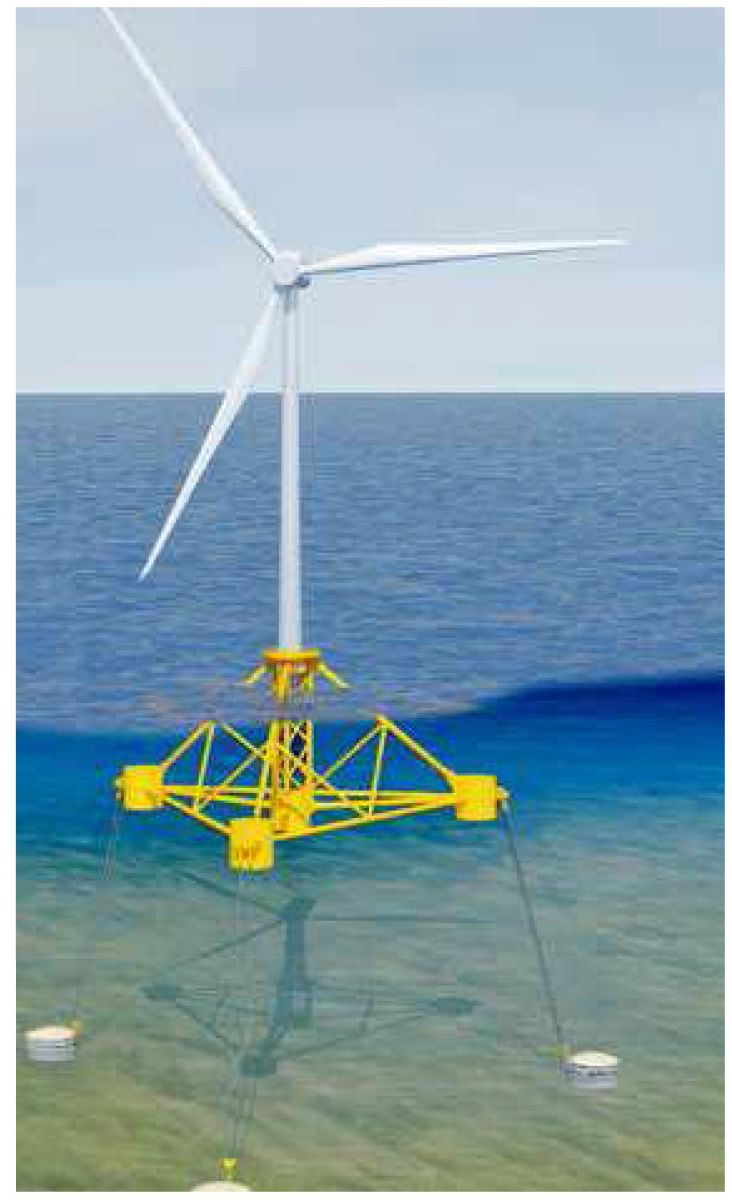

Fig. 6. Eolienne sur ancrage tendu (projet Provence Grand Large). Fig. 6. Wind turbine moored by tension lines (Provence Grand Large project).

- le projet SOLCYP+ qui prolonge les études sur les chargements cycliques conduites lors du projet SOLCYP mais en se focalisant sur le comportement des monopieux de fondations d'éoliennes en mer.

On trouvera réunis dans ce numéro spécial de la RFG les textes des communications présentées lors de cette demijournée. 


\section{Références}

CFMS. 2019. Recommandations pour la conception et le dimensionnement des fondations d'éoliennes offshore. Disponible sur www.cfms-sols.org.

DNVGL-RP-C212. 2017. Offshore soil mechanics and geotechnical engineering, ed. 2007, DNV GL AS, 182 p. Available from www. dnvgl.com.

DNVGL-ST-0126. 2016. Standard-Support structures for wind turbines, ed. 2016, DNV GL AS, 181 p. Available from www. dnvgl.com.

DNVGL-ST-0145. 2015. Standard-Offshore substations, ed. 2016, DNV GL AS, 191 p. Available from www.dnvgl.com.

DNVGL-ST- 0437. 2016. Standard-Loads and site conditions for wind turbines, ed. 2016, DNV GL AS, 107 p. Available from www. dnvgl.com.
Dupla JC, Palix E, Damblans G, et al. 2019. Le projet ANR SOLCYP+ pour améliorer le dimensionnement des monopieux utilisés comme fondations d'éoliennes marines. Revue française de géotechnique 158: 4.

IEC 61400-1. 2019. Wind energy generation systems. Part I: Design requirements. Norme IEC, Webstore IEC ou AFNOR Éditions.

IEC 61400-3. 2009. IEC International Standard. Wind turbines. Part 3: Design requirements for offshore wind turbines, IEC webstore; traduit en français: NF EN 61400-3 Eoliennes. Partie 3 : Exigences de conception des éoliennes en mer. AFNOR Éditions.

Jardine RJ, Kontoe S, Liu TF, et al. 2019. Improving the design of piles driven in chalk through the ALPACA research project. Revue française de géotechnique 158: 2.

NF P 94-262. 2012. National application standard of the Eurocode 7 -Fondations profondes. Norme Française, AFNOR Éditions.

Citation de l'article : Patrick Berthelot, Alain Puech, Françoise Ropers. Les développements éoliens au large des côtes françaises Introduction à la journée technique du 06 décembre 2018. Rev. Fr. Geotech. 2019, 158, 1. 